\title{
RIGHT NUCLEUS IN GENERALIZED RIGHT ALTERNATIVE RINGS
}

K. Jayalakshmi*1, S. Madhavi Latha ${ }^{2}$

${ }^{* 1}$ Dept. of Mathematics, JNTUA University, Ananthapur, A.P., INDIA

${ }^{2}$ Dept. of Mathematics, Sri C.V. Raman Institute of Technology \& Sciences, Tadipatri, A.P., INDIA

*Correspondence Author: kjay.maths@jntua.ac.in

\section{Abstract:}

Some properties of the right nucleus in generalized right alternative rings have been presented in this paper. In a generalized right alternative ring $R$ which is finitely generated or free of locally nilpotent ideals, the right nucleus $\mathrm{Nr}$ equals the center $\mathrm{C}$. Also, if $\mathrm{R}$ is prime and $\mathrm{Nr} \neq$ $C$, then the associator ideal of $R$ is locally nilpotent. Seong Nam [5] studied the properties of the right nucleus in right alternative algebra. He showed that if $R$ is a prime right alternative algebra of char. $\neq 2$ and Right nucleus $N_{r}$ is not equal to the center $C$, then the associator ideal of $R$ is locally nilpotent. But the problem arises when it come with the study of generalized right alternative ring as the ring dose not absorb the right alternative identity. In this paper we consider our ring to be generalized right alternative ring and try to prove the results of Seong Nam [5]. At the end of this paper we give an example to show that the generalized right alternative ring is not right alternative.

Keywords:

Generalized right alternative ring, Right alternative ring, Right nucleus, Nucleus, center, Locally nilpotent.

Cite This Article: K. Jayalakshmi, and S. Madhavi Latha, "Right Nucleus in Generalized Right Alternative Rings." International Journal of Research - Granthaalayah, Vol. 3, No. 1(2015):

1-12. DOI: https://doi.org/10.29121/granthaalayah.v3.i1.2015.3044.

\section{INTRODUCTION}

The studies of varieties of rings like generalized right alternative rings, generalized alternative rings and generalized $(-1,1)$ rings were initiated by Kleinfeld $[1,2,3]$ with the strongest result on the structure of generalized right alternative rings. Smith [4] studied certain generalized right alternative rings with equivalent nilpotencies.

We know that a nonassociative ring $R$ is said to be a generalized right alternative ring if it satisfies the following identities :

$\bar{A}(w, x, y, z)=(w x, y, z)+(w, x,[y, z])=w(x, y, z)+(w, y, z) x, \quad 1$ $(x, x, x)=0$

and $([x, y], y, y)=0$, for all $w, x, y, z$ in $R$.

Throughout this section $R$ is assumed to be generalized right alternative ring with char. $\neq 2$. The right nucleus $N_{r}$, the nucleus $N$ and the centre $C$ are defined as 


\section{INTERNATIONAL JOURNAL of RESEARCH -GRANTHAALAYAH \\ A knowledge Repository}

Science

$N_{r}=\{n \in R /(R, R, n)=0\}-$ right nucleus.

$N=\{n \in R /(n, R, R)=0=(R, n, R)=(R, R, n)\}-$ nucleus.

$C=\{c \in N /[c, N]=0\}-$ center.

We use the following identities which are valid in generalized right alternative ring.

$\bar{B}(w, x, y, z)=(w x, y, z)-(w, x y, z)+(w, x, y z)=w(x, y, z)+(w, x, y) z$.

$[x y, z]-x[y, z]-[x, z] y=(x, y, z)-(x, z, y)+(z, x, y)$.

Substituting $z=n \in N_{r}$ in 4.3.1, we obtain

$(w x, y, n)+(w, x,[y, n])=w(x, y, n)+(w, y, n) x$.

i.e., $(w, x,[y, n])=0$.

i.e., $\left(R, R,\left[R, N_{r}\right]\right)=0$.

Therefore $\left[R, N_{r}\right] \subseteq N_{r}$.

Subtracting 4.3.1 from 4.3.6 we obtain

$\bar{C}(w, x, y, z)=(w, x, z y)-(w, x y, z)-(w, x, y) z+(w, y, z) x=0$.

Now $\bar{A}(a, b, c, x, y)+\bar{A}(a, b, x, y) c-\bar{A}(a, b, c,[x, y])-\bar{A}(a, b c, x, y)$

- $\bar{A} a(b, c, x, y)-\bar{C}(a, b,[x, y], c)$, we obtain

$\bar{D}(a, b, c, x, y)=((a, b, c), x, y)-(a,(b, x, y), c)-(a, b,(c, x, y))$

$-((a, x, y), b, c)+(a, b, c)[x, y]-(a, b, c[x, y])$

$+(a, b,[x, y]) c=0$.

Now with $c \in N_{r}$ in 4.3.10, we obtain

$(a, b,(c, x, y))=-(a, b, c[x, y])+(a, b,[x, y]) c$

$=-(a, b, c[x, y])+(a, b,[x, y] c)$

$=(a, b,[c,[x, y]])$

$=0$.

Hence $(a, b,(c, x, y))=0$ implies $\left(R, R,\left(N_{r}, R, R\right)\right)=0$.

i.e. $\left(N_{r}, R, R\right) \subseteq N_{r}$.

Let us define $\bar{N}_{r}=\left\{n \in N_{r} / n R \subseteq N_{r}\right\}=0$.

$n=z \in N_{r}$ in 4.3.6 implies $(w, x, y n)=(w, x, y) n$.

Linearization of 4.3.2 gives

$\bar{E}[x, y]=(x, y, y)+(y, x, y)+(y, y, x)=0$

\section{RESULT AND DISCUSSIONS}

Lemma 1: Suppose that $m \in N_{r}$ and $x, y, z, w \in R$. Then

(i) $(x, y, z) m=(x, y, z m)=(x, y, m z)=(x, y m, z)$

$=(x m, y, z)-x(m, y, z)$;

(ii) $[x y, m]=x[y, m]+[x, m] y+(m, x, y)-(x, m, y)$;

(iii) $(x, y, z)[m, z]=0$;

(iv) $(x, y, z)(m, w, z)=0$;

(v) If $[m, R]=0$, then $m \in C$.

Proof: To prove $(i)$ we show that

$(x, y, z m)=(x, y, z) m,(x, y, m z)=(x, y, z) m,(x, y m, z)=(x, y, z) m$ and

$(x m$, $y, z)-x(m, y, z)=(x, y, z) m$. 


\section{INTERNATIONAL JOURNAL of RESEARCH -GRANTHAALAYAH \\ A knowledge Repository}

Science

From (13), we obtain $(x, y, z m)=(x, y, z) m$.

From (9), we obtain $(x, y, m z)=(x, y z, m)+(x, y, z) m+(x, z, m) y$.

i.e., $(x, y, m z)=(x, y, z) m$.

Again from (9), we obtain

$(x, y m, z)=(x, y, z m)+(x, y, m) z-(x, m, z) y$

$=-(x y, z, m)+(x, y z, m)+(x, y, z) m-(x, m, z) y \operatorname{using}(6)$

$=(x, y, z) m-(x, m, z) y$.

To show that $(x, m, z) y=0$, we again consider

$(x, y m, z)=(x, y, z m)+(x, y, m) z-(x, m, z) y$

$=(x, y, z m)-(x, m, z) y$

$=(x, y, m z)-(x, m, z) y$

$=-(x y, m, z)+(x, y m, z)+x(y, m, z)+(x, y, m) z$ using $(9)$

$=-(x y, m, z)+(x, y m, z)+(x y, m, z)+(x, y,[m, z])-(x, m, z) y$ using (1).

i.e., $(x, y m, z)=(x, y m, z)-(x, m, z) y$.

i.e., $(x, m, z) y=0$.

Hence $(x, y m, z)=(x, y, z) m$.

Now we show that $(x m, y, z)-x(m, y, z)=(x, y, z) m$.

For, from (6) we have

$(x m, y, z)-x(m, y, z)=(x, m y, z)-(x, m, y z)+(x, m, y) z$.

$$
\begin{aligned}
= & (x, m y, z)-(x, m, y) z+(x, y, z) m-(x, m y, z) \\
& +(x, m, y) z \text { using }(9) . \\
= & (x, y, z) m .
\end{aligned}
$$

Hence $(x m, y, z)-x(m, y, z)=(x, y, z) m$.

(ii) Using (7) with $z=m \in N_{r}$, we obtain

$[x y, m]-x[y, m]-[x, m] y=(x, y, m)-(x, m, y)+(m, x, y)$.

i.e., $[x y, m]=x[y, m]+[x, m] y+(x, m, y)-(m, x, y)$.

(iii) Using 4.3.7 with $x=y=z$ and $z=m \in N_{r}$, we obtain

$\left[z^{2}, m\right]-z[z, m]-[z, m] z-(z, z, m)+(z, m, z)-(m, z, z)=0$.

i.e., $\left[z^{2}, m\right]=z[z, m]+[z, m] z-(z, m, z)+(m, z, z)$

$$
\begin{aligned}
& =z[z, m]+(z m) z-(m z) z-(z m) z+z(m z)+(m, z, z) \\
& =z[z, m]-(m z) z+z(m z)+(m, z, z) \\
& =z[z, m]+[z,(m z)]+(m, z, z),
\end{aligned}
$$

where $\left[z^{2}, m\right] \in N_{r},(m, z, z) \in N_{r}, m z \in \bar{N}_{r}$.

i.e., $[z,(m z)] \in N_{r}$.

Thus $z[z, m] \in N_{r}$.

Hence $(x, y, z)[m, z]=0$.

(iv) $(x, y, z)(m, w, z)=(x, y, z(m, w, z))$

$$
\begin{aligned}
& =(x, y,[z,(m, w, z)])-(x, y,(m, w, z) z) \\
& =-(x, y,(m, w, z) z) .
\end{aligned}
$$

But $(m, w, z) \in N_{r}$.

Hence $(m, w, z) z \in \bar{N}_{r}$, from (12). 


\section{INTERNATIONAL JOURNAL of RESEARCH -GRANTHAALAYAH

Thus $(x, y, z)(m, w, z)=0$.

(v) We use the following identity which is valid in any arbitraty ring.

$(x, y, m)+(y, m, x)+(m, x, y)=[x y, m]+[y m, x]+[m x, y]$

for all $x, y \in R$ and $m \in N_{r}$.

Now using (12) and (8) and the hypothesis, we obtain $[x y, m]=0$,

$[y m, z]=0=[m x, y]$ which implies $(y, m, x)=-(m, x, y)$.

So on rotation we have $(m, x, y)=-(y, m, x)=(x, y, m)=0$.

Hence $m \in C$. Thus we complete the proof of the Lemma.

Let $\tilde{R}$ be the ring obtained by adjoining a 1 to $R$ in the usual way.

We now prove the following Lemmas:

Lemma 2: If $n \in N$, then the ideal generated by $[R, n]$ is

$V_{n}=\tilde{R}[R, n]=[R, n] \tilde{R}$.

Proof: Let $\tilde{R}[R, n]$ be the set of all finite sums

$\sum\left[r_{i}, n\right]+\sum s_{j}\left[t_{\mathrm{j}}, n\right]$.

Since $n \in N$ from Lemma (1), (ii) we obtain,

$[x y, n]=x[y, n]+[x, n] y$, so that the two expressions for $V_{n}$ are equal. Then $R V_{n}=R \tilde{R}[R, n]=$ $\tilde{R}[R, n] \subseteq V_{n}$ and

$V_{n} R=[R, n] \tilde{R} R=[R, n] \widetilde{R} \subseteq V_{n}$.

Hence $V_{n}$ is an ideal of $R$.

Lemma 3: Let $V$ be the ideal of $R$ generated by $\left[R, N_{r}\right]$ and let

$P=\{p \in R ; P V=0\}$. Then

(i) $V=\tilde{R}\left[R, N_{r}\right]=\left[R, N_{r}\right] \tilde{R}$;

(ii) if $p\left[N_{r}, R\right]=0$, then $p \in P$;

(iii) $P$ is an ideal of $R$.

Proof: $(i)$ we have $x n^{\prime}=\left[x, n^{\prime}\right]+n^{\prime} x$.

Now substituting $n^{\prime}=[y, n] \in N_{r}$ in the above equation implies

$x[y, n]=\left[x, n^{\prime}\right]+[y, n] x$.

i.e., $\tilde{R}\left[R, N_{r}\right]=\left[R, N_{r}\right] \tilde{R}$.

Let $r \in R$ and $n \in N_{r}$ then $r(\tilde{R}[r, n])=r \tilde{R}[r, n]-(r, \tilde{R},[r, n])$

Hence $r(\tilde{R}[r, n]) \subseteq \tilde{R}\left[R, N_{r}\right]$.

$$
\subseteq \tilde{R}\left[R, N_{r}\right]
$$

Thus $\tilde{R}\left[R, N_{r}\right]$ is a left ideal and

$\tilde{R}\left[R, N_{r}\right] \cdot R \subseteq R \cdot \tilde{R}\left[R, N_{r}\right]$

$=\left(R, \tilde{R},\left[R, N_{r}\right]\right)-R \tilde{R}\left[R, N_{r}\right]$

$\subseteq \tilde{R} \tilde{R}\left[R, N_{r}\right]$

$=\tilde{R}\left[R, N_{r}\right]$.

Thus $\tilde{R}\left[R, N_{r}\right]$ is the right ideal.

Hence $\tilde{R}\left[R, N_{r}\right]$ is an ideal of $R$.

(ii) we have $0=\left(p, \tilde{R},\left[N_{r}, R\right]\right)$ 


\section{INTERNATIONAL JOURNAL of

$$
=p \tilde{R} \cdot\left[N_{r}, R\right]-p \cdot \tilde{R}\left[N_{r}, R\right]
$$

i.e., $p \tilde{R} \cdot\left[N_{r}, R\right]=p \cdot \tilde{R}\left[N_{r}, R\right]$

But $P V=p \cdot \tilde{R}\left[N_{r}, R\right]$

$$
\subseteq P\left[N_{r}, R\right]=0 .
$$

Hence $p \in P$.

(iii) If $p \in P$ and $r \in R$, then

$0=\left(p, r,\left[N_{r}, R\right]\right)$

$=p r \cdot\left[N_{r}, R\right]-p \cdot r\left[N_{r}, R\right]$

i.e., $p r \cdot\left[N_{r}, R\right]=0$ implies $p r \cdot\left[N_{r}, R\right] \subseteq P V=0$.

And $\left(r, p,\left[N_{r}, R\right]\right)=r p \cdot\left[N_{r}, R\right]-r \cdot p\left[N_{r}, R\right]$.

i.e., $r p \cdot\left[N_{r}, R\right]=r \cdot p\left[N_{r}, R\right]$

$$
=0 \text { using }(i i) \text {. Hence } P \text { is an ideal of } R \text {. }
$$

We know that a any arbitrary ring is purely nonassociative if $R$ is not associative and contains no nonzero ideals in the nucleus $N$. But a generalized right alternative ring $R$ purely nonassociative if the ring is of char. $\neq 2$.

Now we prove the following Lemma:

Lemma 4: Suppose that $R$ is semiprime and purely nonassociative. Then for all $m, n \in N_{r}$ and $x, y$ $\in R$, we have

(i) $[n, x]^{2}=0$;

(ii) $[m, n]=0$;

(iii) $[x, n][x, m]=0$;

(iv) $[x, m][y, m]=0$.

Proof: We first show that $\bar{N}_{r}=\left\{n \in N_{r} / n R \subseteq N_{r}\right\}$ is an ideal of $R$.

For, from (11) with $n \in \bar{N}_{r}, x, y \in R$ we obtain

$0=(s, t,(n, x, y))$

$=(s, t, n x \cdot y)-(s, t, n \cdot x y)$.

i.e., $(s, t, n x \cdot y)=(s, t, n \cdot x y)=0$.

Therefore $n x \cdot y \in N_{r}$.

Thus $n x \in \bar{N}_{r}$.

Hence $\bar{N}_{r} R \subseteq \bar{N}_{r}$.

Now $x n=[x, n]+n x$, then $x n \cdot y=(x, n, y)+x \cdot n y$.

But $x \cdot n y=[x, n y]+n y \cdot x \in N_{r}$.

So $x \cdot n y \in N_{r}$.

Also $(x, n, y)=-(n, y, x)$.

Hence $x n \cdot y=-(n, y, x)+x \cdot n y \in N_{r}$.

Thus $x n \cdot y \in N_{r}$.

Therefore $x n \in \bar{N}_{r}$. i.e., $R \bar{N}_{r} \subseteq \bar{N}_{r}$.

Hence $\bar{N}_{r}=\left\{n \in N_{r} / n R \subseteq N_{r}\right\}$ is an ideal of $R$.

We now show that $P=\left\{p \in R / p \bar{N}_{r}=0\right\}$ is an ideal of $R$. 


\section{INTERNATIONAL JOURNAL of RESEARCH -GRANTHAALAYAH

For, with $p \in P, n \in \bar{N}_{r}$ we have

$y p \cdot n=y \cdot p n=0$.

Therefore $y p \in P$.

i.e., $R P \subseteq P$ and $p y \cdot n=p \cdot y n=0$.

i.e., $p y \in P$.

Hence $P R \subseteq P$. Thus $P$ is an ideal of $R$.

From (6) with $z \in \bar{N}_{r}$, we obtain

$(w x, y, z)-(w, x y, z)+(w, x, y z)=w(x, y, z)+(w, x, y) z$.

Thus we have $(w, x, y) z=0$.

i.e., $(R, R, R) \bar{N}_{r}=0$.

Hence $(R, R, R) \subseteq P$.

Now $\left(P \cap \bar{N}_{r}\right)^{2} \subseteq P \bar{N}_{r}=0$ and semiprimeness gives $P \cap \bar{N}_{r}=0$.

Since $\left(\bar{N}_{r}, R, R\right) \subseteq P \cap \bar{N}_{r}$.

Now $x \in N_{r}$ in (6) gives

$$
\begin{aligned}
(w, x y, z)= & (w x, y, z)+(w, x, y z)-w(x, y, z)-(w, x, y) z \\
= & ((w x) y) z-(w x)(y z)+(w x)(y z)-w(x(y z))-w((x y) z)+w(x(y z)) \\
& -((w x) y) z+(w(x y)) z \\
= & -w((x y) z)+(w(x y)) z .
\end{aligned}
$$

Thus $(w, x y, z)=-(w, x y, z)$.

i.e., $(w, x y, z)+(w, x y, z)=0$.

i.e., $2(w, x y, z)=0$.

Hence by char. $\neq 2$ we obtain $(w, x y, z)=0$.

Thus $\left(R, N_{r} R, R\right)=0$.

Therefore $\left(R, \bar{N}_{r}, R\right)=0$.

Hence $\bar{N}_{r} \subseteq N$.

Therefore by pure nonassociativity $\bar{N}_{r}=0$.

(i) From (6) we obtain

$(w, y, r[m, x][m, x])=(w y, r[m, x],[m, x])+(w, y r[m, x],[m, x])$

$$
+w(y, r[m, x],[m, x])+(w, y, r[m, x])[m, x] .
$$

But $r[m, x] \in N_{r}$ from Lemma 1 (iii).

Therefore we obtain $\left(w, y, r[m, x]^{2}\right)=0$ implies $[m, x]^{2} \in \bar{N}_{r}$.

Hence $[m, x]^{2}=0$.

(ii) From (7), we have $\left[R, N_{r}\right] \subseteq N_{r}$. Now let $[m, n] \in N_{r}$.

From Lemma (1) (iii) $[m, n] r \in N_{r}$.

Hence from (6), we obtain

$0=(w, x, r[m, n])=(w, x, r)[m, n]$.

i.e., $(w, x, r)[m, n]=0$ implies $(R, R, R)\left[N_{r}, N_{r}\right]=0$.

i.e., $A\left[N_{r}, N_{r}\right]=0$.

But $A$ being the associator ideal we have $A \neq 0$ and hence $\left[N_{r}, N_{r}\right]=0$.

Therefore we have $[m, n]=0$.

(iii) Linearization of $(i)$ on $n$ gives 


\section{INTERNATIONAL JOURNAL of RESEARCH -GRANTHAALAYAH

$$
\begin{aligned}
0 & =[m+n, x][m+n, x] \\
& =[m, x][m, x]+[m, x][n, x]+[n, x][m, x]+[n, x][n, x] \\
& =[m, x][n, x]+[n, x][m, x] .
\end{aligned}
$$

Since $N_{r}$ is commutative by $(i i)$ this gives $2[m, x][n, x]=0$.

Hence by char. $\neq 2$, we obtain $[m, x][n, x]=0$.

(iv) Linearization of (i) on $x$ and arguing as in (iii), we obtain

$[n, x][n, y]=0$.

Thus we complete the proof of the Lemma.

Theorem 1: Suppose that $R$ is semiprime and purely nonassociative. Then $N=C$.

Proof: Given $n \in N$. Let $V_{n}$ be as in Lemma 2. Then

$$
\begin{aligned}
V_{n}{ }^{2} & =\tilde{R}[R, n] \cdot[R, n] \tilde{R} \\
& =\tilde{R}[R, n] \cdot \tilde{R}[R, n] \\
& =-(\tilde{R}[R, n], \tilde{R},[R, n])+(\tilde{R}[R, n] \cdot \tilde{R})[R, n] .
\end{aligned}
$$

But $(\tilde{R}[R, n], \tilde{R},[R, n])=0$ implies that

$$
\begin{aligned}
V_{n}^{2} & =(\tilde{R}[R, n] \cdot \tilde{R})[R, n] \\
& =\tilde{R} \tilde{R}[R, n] \cdot[R, n] \\
& =\tilde{R}[R, n] \cdot[R, n] \\
& =(\tilde{R},[R, n],[R, n])+\tilde{R} \cdot[R, n][R, n] \\
& =\tilde{R} \cdot[R, n][R, n] \\
& =0 \text { using Lemma } 4 \text { (iv). }
\end{aligned}
$$

By the semiprimeness $V_{n}=0$.

Hence $n \in C$.

Thus $N \subseteq C$.

Corollary 1: Suppose that $R$ is prime but nonassociative then, $N=C$.

Proof: It is sufficient to show that $R$ is purely nonassociative. Let $I$ be any ideal in the nucleus.

Then $(R, R, R) I=(R, R, R I) \subseteq(R, R, I)=0$.

Thus if $A=\tilde{R}(R, R, R)$ is the associator ideal of $R$, then $A I=(0)$. But $R$ is nonassociative and prime so we have $I=0$. Thus $R$ is purely nonassociative.

From now onwards $R$ is assumed to be semiprime and purely nonassociative.

Lemma 5: If $m \in N_{r}$ and $m\left[N_{r}, R\right]=0$ then $m \in C$. If further $m^{2}=0$, then $m=0$.

Proof: Let $P$ be as in Lemma 3. Then $m \in P$ by Lemma 3 (ii).

So $[m, R] \subseteq P \cap V$. Since $P V=0$ we find as in Lemma 4 that $[m, R]=0$. By Lemma $1(v)$ we obtain $m \in C$. Hence the ideal generated by $m$ is $\tilde{R} m$. If $m^{2}=0$, then $(\tilde{R} m)^{2}=0$, so that $\tilde{R} m=0$. Hence $m=0$.

For a given finite list $M=\left\{a_{1}, \ldots, a_{k}\right\}$ of elements of $R$, we define $T(M)=\left[N_{r}, a_{1}\right] \ldots\left[N_{r}, a_{k}\right]$, i.e., $\left\{\left[m, a_{1}\right] \ldots\left[m, a_{k}\right] ; m_{i} \in N_{r}\right\}$. We note that $T(M) \subseteq N_{r}$. Also, by Lemma 4 (ii) it is zero if it has any repetitions. For the same reason if $t \in T(M)$ then $t^{2}=0$. 


\section{INTERNATIONAL JOURNAL of RESEARCH -GRANTHAALAYAH \\ A knowledge Repository}

Science

We shall allow the empty list $M=\phi$, defining $T(\phi)=1$. (The unit element of $\tilde{R}$ ). In all cases including $M=\phi$ we have $\left[N_{r}, a\right] T(M) \quad=T(M \cup\{a\})$. Next we define, $L(M)=\{w \in R:[w$, $\left.\left.N_{r}\right] T(M)=0\right\}$. In particular, $L(\phi)=\left\{w \in R:\left[w, N_{r}\right]=0\right\}$.

Now we prove the following Lemma:

Lemma 6: ( $i)$ If $b \in L(M)$ then, $\left(N_{r}, b, R\right) T(M)=0$.

(ii) $L(M)$ is a subring of $R$.

Proof: $(i)$ We have $0=\left(N_{r}, R, R\right)\left[b, N_{r}\right] T$

$$
\begin{aligned}
& =\left(N_{r}, b, R\right)\left[R, N_{r}\right] T \text { using Lemma } 1(i i i) \\
& =\left(N_{r}, b, R\right)\left[\left[R, N_{r}\right], T\right]+T\left[R, N_{r}\right] \\
& =\left(N_{r}, b, R\right) T\left[R, N_{r}\right] \text { using Lemma } 4(i i) \\
& =\left(N_{r}, b, R T\right)\left[R, N_{r}\right] \text { using Lemma } 1(i) .
\end{aligned}
$$

Thus if $z \in\left(N_{r}, b, R T\right)$, then $z\left[R, N_{r}\right]=0$. Also $z$ is of the form $(m, b, r)$.

So $z^{2}=0$ by Lemma 1 (iv). Hence $z=0$ by Lemma 5 .

i.e., $0=\left(N_{r}, b, R T\right)=\left(N_{r}, b, R\right) T$.

(ii) Suppose that $x, y \in L(M)$ and $m \in N_{r}$. Then by Lemma 1 (ii) we obtain $[x y, m]=x[y, m]+[x$, $m] y+(m, x, y)-(x, m, y)$.

Since $T \subseteq N_{r}$ we now have

$[x y, m] T \subseteq x[y, m] T+([x, m] y) T+(m, x, y) T-(x, m, y) T$.

Now substituting $[x, m]=m^{\prime}$, we obtain

$[x y, m] T \subseteq x[y, m] T+\left[m^{\prime}, y\right] T+y[x, m] T+(m, x, y) T-(x, m, y) T$.

The first three terms are zero by the assumption and $(m, x, y) T=0$ by Lemma $6(i)$.

To show that $(x, m, y) T=0$, we use equation (6)

$(x m, y, T)-(x, m y, T)+(x, m, y T)=x(m, y, T)+(x, m, y) T$.

But $T=\left[N_{r}, a\right] \subseteq N_{r}$. Hence we obtain $(x, m, y T)=(x, m, y) T$ which implies $\left(x, m, y\left[N_{r}, a\right]\right)=(x, m$, $y)\left[N_{r}, a\right]$. Hence $\left(x, m, y\left[N_{r}, a\right]\right)=0$ since $y \in L(M)$. Thus $(x, m, y)\left[N_{r}, a\right]=0$. That is $(x, m, y) T=$ 0 and since $m \in N_{r}$ was arbitrary. This shows that $\left[x y, N_{r}\right] T=0$. Thus $x y \in L$. Therefore $L(M)$ is a subring of $R$.

We say that $R$ is finitely generated $\bmod N_{r}$ if there is a finite subset $M$ of $R$ such that the subring of $R$ generated by $N_{r} \cup M$ is all of $R$.

We now prove the following Theorem:

Theorem 2: Suppose that $R$ is semiprime purely nonassociative and is finitely generated $\bmod N_{r}$. Then $N_{r}=C$.

Proof: Suppose that $R$ is generated by $N_{r} \cup M$, where $M=\left\{a_{1}, \ldots, a_{k}\right\}$ we will show that if $S$ is any list of terms from $M$, then $L(S)=R$ and provided that $S \neq \phi, T(S)=0$.

We do so by reverse induction on the length $r=|S|$ of $S$. If $|S|=k+1$ then $S$ has repetitions, so that $T(S)=0$. Hence clearly $L(\mathrm{~S})=R$. Suppose that we have both results for list of length $r+1$, and $S$ is the list of length $r$. Then for $a \in M$ we have $\left[a, N_{r}\right] T(S)=T(S)$, where $S^{\prime}=S \cup\{a\}$ has 


\section{INTERNATIONAL JOURNAL of RESEARCH -GRANTHAALAYAH

length $r$. Thus $\left[a, N_{r}\right] T(S)=0$. So $a \in L(S)$. Hence $M \subseteq L(S)$. As $\left[N_{r}, N_{r}\right]=0$ by Lemma 4 (ii), we also have $N_{r} \subseteq L(S)$. Thus by Lemma $6(i i), L(S)$ is a subring of $R$ containing $M \cup N_{r}$. That is $L(S)$ $=R$. Next suppose that $S \neq \phi$, and $t \in T(S)$. Since $L(S)=R$, we have $\left[R, N_{r}\right] T(S)=0$. Hence $t\left[R, N_{r}\right]$ $=0$. Also we have seen that $t^{2}=0$. So by Lemma 5 we have $t=0$. That is $T(S)=0$. This concludes the induction. Hence $L(\phi)=R$ gives $\left[R, N_{r}\right]=0$. Thus $N_{r}=C$ by Lemma $1(v)$.

Theorem 3: Suppose that $R$ is purely nonassociative and free of locally nilpotent ideals. Then $N_{r}$ $=C$.

Proof: From Lemma 4 (ii) $[m, n]=0$, where $m, n \in N_{r}$ implies $N_{r}$ is commutative. But $\left(N_{r}, R, R\right)$ $\subseteq N_{r}$ from 4.3.11 and $\left[N_{r}, R\right] \subseteq N_{r}$ from (8). Now if $N_{r}$ is contained in $N_{\beta}$ the right alternative nucleus which is defined by $N_{\beta}=\left\{n \in R /(x, x, n)=\left(x^{2}, x, n\right)=\left(x^{2}, y, n\right)+(x \circ y, x, n)=0 \forall x\right.$, $y, \in R\}$ and $\left(N_{r}, N_{r}, R\right)=0$. Using the identity (14) with $b \in N_{r}$ we have $(a, b, b)=0,(b, a, b)=0$ and so $(b, b, a)=0$. That is $\left(N_{r}, N_{r}, R\right)=0$. Thus if we let $I$ be the nil radical of $N_{r}$, then $I+I R$ is locally nilpotent ideal of $R$ such that $\left(N_{r}, R, R\right),\left[N_{r}, R\right] \subseteq I$. Since $R$ is free of locally nilpotent ideals $\left(N_{r}, N_{r}, R\right)=0$ and $\left[N_{r}, R\right]=0$. And from Lemma $1(v)$ we obtain $N_{r}=C$. Thus we complete the proof of the Theorem.

We consider the prime rings in which $N_{r} \neq C$ (supposing such exist). In such a ring the ideal $V$ of Lemma 3 is nonzero by Lemma $1(v)$. Hence the ideal $P$ is zero. Thus by Lemma $3(i i)$, if $p\left[N_{r}, R\right]$ $=0$ then $p=0$. We assume that $R$ has this later property (and do not appeal directly to the primeness).

We now prove the following Lemmas:

Lemma 7: If $u$ is of the form $(z, w, b)$ or $y(z, w, b)$ then, $(x, b, u)=0$.

Proof: If $m \in N_{r}$ then from (10), we obtain

$((x, b, u), r, m)-(x,(b, r, m), u)-(x, b,(u, r, m))-((x, r, m), b, u)$

$+(x, b, u)[r, m]-(x, b, u[r, m])+(x, b,[r, m]) u=0$.

We have $((x, b, u), r, m),(x,(b, r, m), u),(x, b,(u, r, m)),((x, r, m), b, u)$,

$(x, b,[r, m]) u$ are all equal to zero since $m \in N_{r}$.

But $(x, b, u[r, m])=0$ since $u[r, m] \in \bar{N}_{r}$.

Thus we have $(x, b, u)[r, m]=0$.

i.e., $(x, b, u)\left[R, N_{r}\right]=0$.

Hence we have $(x, b, u)=0$.

Lemma 8: If $X \subseteq R$ then,

(i) the left ideal of $R$ generated by $(R, X, R)$ is $\tilde{R}(R, X, R)$;

(ii) the left ideal of $R$ generated by $X$ is $L_{X}=\tilde{R} X+\tilde{R}(R, X, R)$;

Proof: (i) we have $r \cdot s(a, x, b)=r s \cdot(a, x, b)-(r, s,(a, x, b))$. Substituting $r s=r^{\prime}$, we obtain $r \cdot s(a, x, b)=r^{\prime} \cdot(a, x, b)-(r, x,(a, s, b))$.

By Lemma 7 it is in $\tilde{R}(R, X, R)$.

(ii) This is now obvious from $R \cdot \tilde{R} X \subseteq R X+(R, R, X)$ and part (i). 


\section{INTERNATIONAL JOURNAL of RESEARCH -GRANTHAALAYAH

Given $a, b \in R$ we will write $L_{a}$ for $\tilde{R}(R, R, a)=L(R, R, a)$ and $L_{a, b}$ for $L(R, a, b)$. Clearly $L_{a, b} \subseteq$ $L_{a}$.

Lemma 9: (i) $L_{a}\left[N_{r}, a\right]=0$;

(ii) $L_{a, b}\left[N_{r}, R\right] \subseteq L_{b}\left[N_{r}, a\right]$;

(iii) $\left(R, L_{b}, b\right)=0$.

Proof: (i) $L_{a}\left[N_{r}, a\right]=\tilde{R}(R, R, a)\left[N_{r}, a\right]$ $=0$ using Lemma 1 (iii).

(ii) $(R, a, b)\left[N_{r}, R\right]=(R, R, b)\left[N_{r}, a\right]$

$$
\subseteq L_{b}\left[N_{r}, a\right] \text {. }
$$

Now set $U=(R, R,(R, a, b))$.

Then $U\left[N_{r}, R\right]=\left(R, R,(R, a, b)\left[N_{r}, R\right]\right)$

$$
\begin{aligned}
& \subseteq\left(R, R, L_{a, b}\left[N_{r}, R\right]\right) \\
& \subseteq\left(R, R, L_{b}\left[N_{r}, a\right]\right) \\
& =\left(R, R, L_{b}\right)\left[N_{r}, a\right] \\
& \subseteq L_{b}\left[N_{r}, a\right] .
\end{aligned}
$$

Hence $L_{b}$ is a left ideal.

Now $L_{a, b}\left[N_{r}, R\right]=\tilde{R}((R, a, b)+U)\left[N_{r}, R\right]$

$$
\begin{aligned}
& =\tilde{R}((R, a, b)+(R, R,(R, a, b)))\left[N_{r}, R\right] \\
& =\tilde{R}\left((R, a, b)\left[N_{r}, R\right]+(R, R,(R, a, b))\left[N_{r}, R\right]\right) \\
& =\tilde{R} L_{a, b}\left[N_{r}, R\right]+\tilde{R}\left(R, R, L_{a, b}\right)\left[N_{r}, R\right] \\
& \subseteq \tilde{R} L_{b}\left[N_{r}, a\right]=L_{b}\left[N_{r}, a\right] .
\end{aligned}
$$

(iii) From Lemma 7 we have $\left(R, L_{b}, b\right)=0$ where $L_{b}=\tilde{R}(R, R, b)$.

Lemma 10: $(i)\left(L_{a, b}, R, L_{a, b}\right)=0$;

$$
\text { (ii) } L_{a, b}^{2}=0 \text {. }
$$

Proof: Using Lemma 1 (i) we have

$\left(L_{a, b}, R, L_{a, b}\right)\left[N_{r}, R\right]=\left(L_{a, b}, R, L_{a, b}\left[N_{r}, R\right]\right)$

$$
\begin{aligned}
& \subseteq\left(L_{a, b}, R, L_{b}\left[N_{r}, a\right]\right) \text { using Lemma } 9(i i) \\
& \subseteq\left(L_{a, b}\left[N_{r}, a\right], R, L_{b}\right)-L_{a, b}\left(\left[N_{r}, a\right], R, L_{b}\right)
\end{aligned}
$$

which is obtained by using Lemma $1(i)$. The first term is zero by Lemma $9(i)$. The second term is contained in $L_{b}\left(N_{r}, R, L_{b}\right)=\tilde{R}(R, R, b)\left(N_{r}, R, L_{b}\right)$ since $\left(N_{r}, R, R\right) \subseteq N_{r}$. But $(R, R, b)\left(N_{r}, R, L_{b}\right)=$ $(R, R, R)\left(N_{r}, b, L_{b}\right)$ is obtained using Lemma $1(i v)$ and this is equal to zero using Lemma 9 (iii). Thus we have $\left(L_{a, b}, R, L_{a, b}\right)\left[N_{r}, R\right]=0$.

(ii) Using Lemma 9 (ii) we have

$L_{a, b} L_{a, b}\left[N_{r}, R\right] \subseteq L_{a, b} L_{b}\left[N_{r}, a\right]$

$$
\subseteq L_{a, b}\left[N_{r}, a\right] L_{b}+L_{a, b}\left[L_{b},\left[N_{r}, a\right]\right]
$$

The first term is zero by Lemma $9(i)$.

The second term is contained in

$$
\begin{aligned}
L_{b}\left[L_{b}, N_{r}\right] & =R(R, R, b)\left[L_{b}, N_{r}\right] \\
& =R\left(R, L_{b}, b\right)\left[R, N_{r}\right] \text { by Lemma } 1(i i i)
\end{aligned}
$$




\section{INTERNATIONAL JOURNAL of RESEARCH -GRANTHAALAYAH

$$
=0 \text { using Lemma } 9 \text { (ii). }
$$

Hence $L_{a, b}^{2}\left[N_{r}, R\right]=0$.

Thus $L_{a, b}^{2}=0$.

Suppose now that we are given finitely many left ideals $X_{i}=L_{a i} b i$.

Lemma 11: Suppose that $c_{1} \ldots c_{k}$ are such that each $c_{i}$ is in some $X_{j}$, and $p$ is the left associated product $c_{1} \ldots c_{k}$. Then

(i) either $p=0$ or every $c_{i}$ is in a different $X_{j}$;

(ii) if some $c_{i}$ is in $X_{j}$, then $p$ is in $X_{j} \tilde{R}$.

Proof: We shall prove the Lemma by using the induction on $k$. If $k=1$ both conclusions are trivial. Suppose that the Lemma is proved for $k-1$, and write $p=c_{1} q$. If $q=0$, then $p=0$ and both the conclusions hold. Suppose then that $q \neq 0$.

(i) By induction hypothesis, $q$ has all $c_{i}$ in different $X_{j}$. If $c_{1}$ is in a new $X_{j}$, then $c_{1} \in X_{j}$, then $p$ is of the required type.

Suppose then that $c_{1} \in X_{j}$ and some $c_{i}$ of $q$ is also in $X_{j}$. By induction hypothesis $q=X_{j} \tilde{R}$.

Hence $p \in X_{j} \cdot X_{j} \tilde{R}$

$$
\begin{aligned}
& =X_{j}^{2} \tilde{R} \text { using Lemma } 10(i) \\
& =0 \text { using Lemma } 10(i i) .
\end{aligned}
$$

(ii) If $c_{1} \in X_{j}$ then certainly $p=c_{1} q \in X_{j} \tilde{R}$. If $c_{i} \in X_{j}$ for some $i>1$, then by inductive hypothesis $q \in X_{j} \tilde{R}$. But it is easily verified that if $L$ is a left ideal of $R$ then so is $L \tilde{R}$. Thus $X_{j} \tilde{R}$ is a left ideal. Hence $p \in X_{j} \tilde{R}$.

The left power of a ring $X$ are defined by $X^{[1]}=X$ and $X^{[n+1]}=X \cdot X^{[n]}$. The ring $X$ is left nilpotent if for some $n$ we have $X^{[n]}=0$.

Lemma 12: Suppose that $R$ is prime and $N_{r} \neq C$. Then any finite generated left ideal of $R$ contained in the asociator ideal $A=\tilde{R}(R, R, R)$ is left nilpotent.

Proof: Suppose that $p \in A$. Then $p$ is a linear combination of terms of the form $(r, a, b)$ and $s(r, a$, $b$ ) for various $a, b \in R$ and $r, s \in R$. The left ideal of $R$ generated by $p$ is thus contained in the left ideal generated by all such terms. But the left ideal generated by $(r, a, b)$ or by $s(r, a, b)$ is contained in $L_{a, b}$. Hence the left ideal generated by $p$ is contained in a finite sum $S_{p}=\sum_{j} L_{a j, b j}$ say. Thus to show that $Q$ is left nilpotent it suffices to show that any left ideal of the form $X=\sum_{k=1}^{m} X_{k}$ is nilpotent, where $X_{k}=L_{a k, b k}$ for some $a_{k}, b_{k} \in R$. But from Lemma 11 (i) that any left-associated product of length $m+1$, with each term in some $X_{k}$ is zero. Since every element of $X^{[m+1]}$ is a linear combination of terms of the type, we have $X^{[m+1]}=0$. Thus $X$ is left nilpotent, as required.

Theorem 4: Suppose that $R$ is prime and $N_{r} \neq C$. Then any finite generated subring of the asociator ideal is left nilpotent.

Proof: Suppose that $p$ is a finite subset of $M$, and $S$ is a subring it generates. The left ideal $Q$ of $R$ generated by $p$ is left nilpotent by Lemma 12 . Since $S \subseteq Q$ we conclude that $S$ is left nilpotent. But 


\section{INTERNATIONAL JOURNAL of RESEARCH -GRANTHAALAYAH \\ A knowledge Repository}

Science

now a result of Slinko [6] shows that $S$, being left nilpotent and finitely generated, is infact nilpotent. Thus we complete the proof of the Theorem.

We give an example of a generalized right alternative ring which shows that generalized right alternative ring is not right alternative.

Example: If $R$ is an associative and commutative ring with an element $1 / 2$, and $M$ is any module over $R$, then $S=R \times M$ can be made into a generalized right alternative ring by the following definition of addition and multiplication. Addition is coordinate wise and multiplication is given by $[a, m]\left[a^{\prime}, m^{\prime}\right]=\left[a a^{\prime}, 1 / 2 a m^{\prime}+1 / 2 a^{\prime} m\right]$. If we identify $M$ by $\{0\} \times M$, then $M$ is a two-sided ideal of $S$, and $[S, M]=0$. If $1 / 2+1 / 2=e(e$ is the identity of $R$ ) then $e$ is an idempotent of $S$, and $-4(m$, $e, e)=m$ for all $m \in M$. There exist generalized right alternative ring which are not right alternative.

\section{CONCLUSIONS}

The ring defined by $\tilde{R}$ which is obtained by adjoining a 1 to $R$ in the usual way leads $V_{n}$ which is defined as $V_{n}=\tilde{R}[R, n]=[R, n] \tilde{R}$ to an ideal. Not only $V_{n}$ but also makes $P$ an ideal defined which is defined by $P=\{p \in R ; P V=0\}$. In a semiprime and purely nonassociative generalized right alternative ring the nucleus equals the center only if $\bar{N}_{r}=\left\{n \in N_{r} / n R \subseteq N_{r}\right\}$ is an ideal of the ring. An additional condition on $R$ is produce which ensures not merely that $N=C$ (as in Theorem 1) but the stronger result $N_{r}=C$.

\section{REFERENCES}

[1] Kleinfeld, E. Generalization of alternative rings-I, Journal of Algebra, 18 (1971), 304-325.

[2] Kleinfeld, E. Generalization of alternative rings, Journal of Algebra, 27 (1973), 604-624.

[3] Kleinfeld, E. A generalization of (-1, 1) rings, Pacific Journal of Math., 53 (1974), 195-202.

[4] Seong Nam, Ng., Right nucleus in Right alternative algebras, Journal of London Mathematical Society, (2), 21 (1980), 456-464.

[5] Smith, H. F. Equivalence nilpotencies in certain generalized right alternative rings, Pacific Journal of Mathematics, Vol. 98. No.2, (1982), 459-467.

[6] Slinko, A. M. On equivalence of certain type of nilpotence in right alternative rings, Algebra $i$ Logika, 9 (1970), 342, 348. 